\title{
State of knowledge of the tachinid fauna of Eastern Asia, with new data from North Korea. Part V. Exoristinae
}

\author{
Agnieszka DRABER-MOŃKO \\ Museum and Institute of Zoology,PAS, Wilcza 64,00-679Warszawa; e-mail: draber@miiz.waw.pl
}

\begin{abstract}
The present paper is a continuation of earlier papers concerning the Tachinidae collected in North Korea by six expeditions of researchers from the Institute of Zoology PAS, Warsaw, Poland. Twenty four species representing 20 genera of the subfamily Exoristinae have been identified in the material collected. Eighteen species are reported in the fauna of Korea for the first time..
\end{abstract}

Key words: Diptera, Tachinidae, Exoristinae, new data, North Korea

\section{INTRODUCTION}

The first part of this paper (Draber-Mońko 2008) was concerned with the Tachinidae belonging to the subfamily Phasiinae. The second part (Draber-Mońko 2011) was concerned with the Tachinidae belonging to the subfamily Tachininae. The third part paper (DraberMońko 2012) was a supplement to the first part. The fourth part (Draber-Mońko 2015) was concerned with the Tachinidae belonging to the subfamily Dexiinae.

The present paper is devoted to the tachinid species belonging to the subfamily Exoristinae. The Exoristinae are the largest subfamily with over 700 species recorded from the Palaearctic Region (Herting \& Dely-Draskovits 1993, Ziegler \& Shima 1996, Richter 2004, Han et al. 2014, Shima 2014), over 280 species from the Russian Far East (Ziegler \& Shima 1996, Richter 2004), over 280 species from Japan (Shima 2014), more than 570 species from China (O’Hara, Shima \& Zhang 2009), over 590 species from the Nearctic Region (O'Hara \& Wood 2004), more than 1320 species from Neotropical (Guimarães 1971) and over 380 species from Oriental Region (Crosskey 1977).

Dipterists subdivide the subfamily Exoristinae into six or more, even several dozen tribes. In the Palaearctic Region these are: Exoristini, Blondeliini, ${ }^{*}$ Acemyini, *Ethillini, Winthemiini, Eryciini, Goniini (Herting \& Dely-Draskovits 1993, Richter 2004). Those marked with an asterisk are not recorded from the Korean Peninsula.

Lepidoptera, Coleoptera and Hymenoptera are most frequent hosts of Exoristinae species, but Dermaptera, Manthodea, Orthoptera and Phasmiida are also known as hosts.

Species of the tribe Exoristini are all oviparous and they deposit unincubated eggs with a hard planoconvex chorion directly on the hosts exoskeleton. Flies of the tribe Blondeliini are divergent in ovipositing habits. They are oviparous or ovolarviparous directly depositing on the hosts exoskeleton, or haemocoel or in the vicinity of their hosts. Species of the tribe Ethillini are all oviparous. Females lay unincubated eggs directly of the hosts exoskeleton. Flies of the tribe Winthemiini are oviparous and females lay unincubated eggs with a hard planoconvex chorion on the exoskeleton of host larvae. Species of the tribe Eryciini are oviparous or ovolarviparous. In both cases eggs are deposited directly of the hosts exoskeleton. Flies of the tribe Goniini are all micro-ovolarviparous. Females lay incubated micotype eggs $(0.2 \mathrm{~mm}$ or less in length) with a hard chorion on the host plants of host insects (Shima 1999). 


\section{MATERIAL AND METODS}

The Exoristinae flies were collected during six expeditions in North Korea: in 1959, 1965, 1966, 1970, 1987 and 1990. Detailed descriptions of these expeditions are in Mroczkowski (1972) and in Bańkowska \& Sterzyńska (1997). The locations of the sampling areas in North Korea were presented previously (Draber-Mońko 2015: Fig. 1).

The scanty material of the Exoristinae, considered here, includes 31 specimens collected with a sweeping net or by using Moericke's yellow traps placed on grass and in brushwood.

The current list of Exoristinae flies from Korea has been supplemented with the species mentioned by other authors: Aldrich 1928, Nakayama 1929, Doi 1938, Shima 1968, 1980, 1983, 1984, 1996, 2006, 2014, Kim \& Kim 1971, Kim \& Kim 1972, Kim \& Nam 1978, Lee \& Kwon 1981, Kim 1981, Hirashima 1989, Fan 1992, Pemberton et al. 1993, Park et al. 1998, Chao \& Xue 1998, Paek et al. 2010, Lee et Han 2007, 2008, 2010, Liu et al.1998, Han et al. 2004, O’Hara, Shima \& Zhang 2009, Byun \& Han 2009, 2011, Byun 2010, Cerretti et Shima 2011, and Han et al. 2014.

The systematic arrangement of the present paper follows that provided by Tschorsnig \& Richter 1998, Richter 2004, O’Hara, Shima \& Zhang 2009.

The general distribution data are given after: Herting \& Dely-Draskovits 1993, Richter 2004 and O'Hara, Shima \& Zhang 2009.

The specimens identified are deposited in the Museum and Institute of Zoology PAS (Warsaw). The species new to Korea have been marked with an asterisk $\left(^{*}\right)$.

\section{SYSTEMATIC REVIEW OF SPECIES}

Subfamily Exoristinae

Tribe Blondeliini

Blondelia Robineau-Desvoidy, 1830

\section{Blondelia nigripes (Fallén, 1810)}

Tachina nigripes Fallén, 1810: 283. Type locality: Sweden.

Distribution: Palaearctic: Europe (all), Transcaucasia, Russia (W. Russia, W. and E. Siberia, S.Far East (Primorsk Territory), Sakhalin, South Kuril Islands (Kunashir, Shikotan) (Richter 2004), M. East, C. Asia, Mongolia, China Provinces: Heilongjang, Jilin, Liaoning, Inner Mongolia, Hebei, Beijing, Shanxi, Gansu, Qinghai, Ningxia Huizu A.R., Xinjiang Uygur A.R., Shanghai, Sichuan, Shaanxi, Xizang A.R., Japan (Hokkaidō, Honshū, Shikoku, Kyūshū) (O’Hara, Shima \& Zhang 2009), Korea (Shima 1984); Oriental: China (Yunnan Prov.) (O’Hara, Shima \& Zhang 2009).

Compsilura Bouché, 1834

\section{Compsilura concinnata (Meigen, 1824)}

Tachina concinnata Meigen, 1824: 412. Type locality: not given (probably Hamburg,Germany).

Material examined: North Korea, Chǒngdžin-si Prov., Kjǒngsǒng distr., Onpho-ri ad Chǒngdžin, 14. 08. 1959, 1 female, leg. B. Pisarski and J. Prószyński.

Distribution: Palaearctic: Europe (all), Transcaucasia, Russia (W. Russia, S. Siberia (Richter 2004), M. East, C. Asia, China (distributed almost all over the country, but has not 
been recorded from Provices: Gansu, Henan, Hong Kong S.A.R., Hubei, Macao S.A.R., Qinghai, Shaanxi, Xinjiang Uygur A.R., Japan (Hokkaidō, Honshū, Shikoku, Kyūshū, Tsushima Is.) (O’Hara, Shima \& Zhang 2009, Shima 2014), Korea (Kim \& Nam 1978, Byun 2010); Oriental: Japan (Ryukyu Is.), Taiwan, China Provinces: Fujian, Guangdong, Guangxi Zhuangzu A.R., Guizhou, Hainan, Hunan, Jiangxi, Yunnan, Zhejiang, India, Indonesia (Jawa, Sulawesi), Malaysia (Pen. Malaysia, E. Malaysia), Nepal, Philippines, Thailand; Australasian: Australia, Papua N.G.; Afrotropical: widespread; Nearctic: introduced and widespread in northeast, also British Columbia to California (O’Hara, Shima \& Zhang 2009).

Compsiluroides Mesnil, 1953

\section{Compsiluroides flavipalpis Mesnil, 1957*}

Compsiluroides flavipalpis Mesnil, 1957: 22. Type locality: Japan, Hokkaido, Obihiro.

Material examined: North Korea, N. Hamgyong Prov., Chǒngdžin-City, Kyowon-ri, 16. 06. 1990, 1 male, leg. E. Chudzicka, E. Kierych and R. Pisarska.

Distribution: Palaearctic: Russia S. Far East (Primorsk Territory), South Kuril Islands (Kunashir) (Richter 2004), Japan (Hokkaidō, Honshū, Shikoku, Kyūshū), China Provinces (Sichuan, Shaanxi); Oriental: China Provinces (Guangdong, Guizhou, Yunnan) (O’Hara, Shima \& Zhang 2009). First record from Korea.

Drinomyia Mesnil, 1962

\section{Drinomyia hokkaidensis (Baranov, 1935)}

Vibrissina hokkaidensis Baranov, 1935: 554. Type locality: Japan, Hokkaido, Sapporo.

Oswaldia bicoloripes Mesnil, 1957: 23. Type locality: Japan, Hokkaido, Obihiro.

Distribution: Palaearctic: Russia S. Far East (Primorsk Territory), South Kuril Islands (Kunashir) (Richter 2004), Japan (Chishima Is., Hokkaidō, Honshū, Kyūshū, Tsushima Is.) (Shima 2014), Korea (Shima 1980, Paek et al., 2010), China Provinces (Beijing, Guizhou, Hebei, Liaoning, Inner Mongolia, Shaanxi, Shanxi, Tianjin, Tibet) (O’Hara, Shima \& Zhang 2009)

Istochaeta Rondani, 1859

\section{Istochaeta aldrichi (Mesnil, 1953)}

Hyperecteina aldrichi Mesnil, 1953: 50. Type locality: Japan, Honshū.

Distribution: Palaearctic: Russia S. Far East, South Kuril Islands (Kunashir) (Richter 2004), Japan (Hokkaidō, Honshū, Shikoku, Kyūshū, Chishima Is.) (Shima 2014), Korea (Hirashima 1989, Paek et al., 2010); Oriental: Taiwan; Nearctic: introduced and established in New York and Massachussets to District of Columbia (O’Hara, Shima \& Zhang 2009).

\section{Istochaeta unicolor (Aldrich, 1928)}

Centeter unicolor Aldrich,1928: 7. Type locality: Korea, Suigen.

Distribution: Palaearctic: Russian S. Far East (Primorsk Territory) (Richter 2004), Korea (Aldrich 1928, Byun 2010). 
Lixophaga Townsend, 1908

\section{Lixophaga cinctella (Mesnil, 1957)}

Lomatacantha cinctella Mesnil, 1957: 24. Type locality: Japan, Hokkaido, Obihiro.

Distribution: Palaearctic: Japan (Hokkaido, Honshu) (Shima 2014), Korea (Byun \& Han 2011), China (Sichuan Prov.) (O’Hara, Shima \& Zhang 2009).

\section{Lixophaga latigena Shima, 1979}

Lixophaga latigena Shima, 1979: 308. Type locality: Japan, Ryukyu Islands, Toku-no-shima, Asahigaoka.

Distribution: Palaearctic: Japan (Honshu, Kyushu) (Shima 2014), Korea (Byun and Han 2011), China Provinces (Anhui, Liaoning, Tibet) (O'Hara, Shima \& Zhang 2009); Oriental: China Provinces (Guangxi Zhuangzu A.R., Yunnan), Japan (Ryukyu Islands) (Shima 2014, O’Hara, Shima \& Zhang 2009).

Medina Robineau-Desvoidy, 1830

\section{Medina luctuosa (Meigen, 1824)}

Tachina luctuosa Meigen, 1824: 347. Type locality: not given (probably Germany, Stolberg).

Distribution: Palaearctic: Europe (all), Russia: European Territory, E. Siberia (Chita Prov.), S. Far East (Amur Prov., Primorsk Territory, South Kuril Islands (Kunashir) (Richter 2004), Japan (Hokkaido, Honshu) (Shima 2014), Korea (Park et al. 1996, Byun 2010), China: Liaoning Prov.,Transcaucasia; Oriental: China Provinces (Guangdong, Yunnan) (O’Hara, Shima \& Zhang 2009).

\section{Medina separata (Meigen, 1824)*}

Tachina separata Meigen, 1824: 406. Type locality: not given (probably Germany, Kiel).

Material examined: North Korea, Kangvǒn-do Prov., Kūmgang-san Mts., Onjong-ri near Kūmgang-san hotel, 23-25. 06. 1990, 2 females, leg. E. Chudzicka, E. Kierych and R. Pisarska.

Distribution: Palaearctic: Europe (all), Russia: E. Siberia (Chita Prov.), Far East (Primorsk Territory) (Richter 2004), Japan (Hokkaido, Honshu), China (Shanxi Prov.) (O’Hara, Shima \& Zhang 2009). First record from Korea.

Meigenia Robineau-Desvoidy, 1830

\section{Meigenia tridentata Mesnil, 1961*}

Meigenia tridentata Mesnil, 1961: 703. Type locality: China, Heilongjiang, Datudinzsa.

Material examined: North Korea, Chǒngdžin-si Prov., Kjǒngsǒng distr., Onpho-ri ad Chǒngdžin, 16. 08. 1959, 1 male, leg. B. Pisarski and J. Prószyński.

Distribution: Palaearctic: Russia: Far East (Amur Prov., Khabarovsk Territory and Primorsk Territory) (Richter 2004), China Provinces: Beijing M., Heilongjiang, Hubei, Liaoning, Sichuan, Shaanxi, Shanxi, Tibet; Oriental: China Provinces: Guangxi Zhuangzu A.R., Guizhou, Hunan, Jiangxi, Yunnan, Zhejiang) (O’Hara, Shima \& Zhang 2009). First record from Korea. 
Metadrinomyia Shima, 1980

Metadrinomyia flavifrons Byu et Han, 2009

Metadrinomyia flavifrons Byu et Han, 2009: 57. Type locality: South Korea.

Distribution: Palaearctic: Korea (Byu et Han, 2009, Paek et al., 2010).

\section{Metadrinomyia proclinata Shima, 1980}

Metadrinomyia proclinata Shima, 1980: 261. Type locality: Japan, Tsushima.

Distribution: Palaearctic: Russia: Far East (Primorsk Territory) (Richter 2004), Japan (Tsushima Island) (Shima 2014), Korean Peninsula (Shima 1980, Byu et Han, 2009, Paek et al., 2010).

\section{Metadrinomyia xanthokolos Byu et Han, 2009}

Metadrinomyia xanthokolos Byu et Han, 2009: 61. Type locality: South Korea.

Distribution: Palaearctic: Korea (Byu et Han, 2009, Paek et al., 2010).

Oswaldia Robineau-Desvoidy, 1863

\section{Oswaldia hirsuta Mesnil, 1970}

Oswaldia hirsuta Mesnil, 1970: 115. Type locality: Japan, Hokkaido, Nukabira.

Distribution: Palaearctic: Japan (Hokkaidō, Honshū, Kyūshū) (Shima 2014), Korea (Hirashima 1989, Paek et al., 2010), China: Heilongjiang Prov., Liaoning Prov. (O'Hara, Shima \& Zhang 2009).

\section{Oswaldia muscaria (Fallén, 1810)*}

Tachina muscaria Fallén, 1810: 272. Type locality: Sweden.

Material examined: North Korea, Chǒngdžin-si Prov., Purjǒng distr., Musu-ri, ad river Susǒng-čhǒn, 1. 06. 1965, 1 female, leg. M. Mroczkowski and A. Riedel.

Distribution: Palaearctic: Europe (all), Russia: Far East (Khabarovsk Territory, Primorsk Territory, Sakhalin, South Kuril Islands (Kunashir) (Richter 2004), Japan (Hokkaido, Honshu, Shikoku, Kyushu) (Shima 2014), Taiwan, China: Heilongjiang Prov., Liaoning Prov.; Oriental: China: Yunnan Prov. (O’Hara, Shima \& Zhang 2009). First record from Korea.

Prodegeeria Brauer et Bergenstamm, 1894

\section{Prodegeeria japonica (Mesnil, 1957)}

Promedina japonica Mesnil, 1957: 26. Type locality: Japan, Hokkaido, Obihiro.

Distribution: Palaearctic: Russia: Far East (Khabarovsk Territory, Primorsk Territory, Sakhalin, South Kuril Islands (Kunashir, Shikotan) (Richter 2004), Japan (Hokkaido, Honshu, Shikoku, Kyushu) (Shima 2014), S. Korea (Hirashima 1989, Herting \& Dely-Draskovits 1993, Paek et al. 2010), China Provinces (Beijing M., Jilin, Liaoning, Sichuan, Shaanxi); Oriental: China Provinces (Guangdong, Hunan, Yunnan, Zhejiang) (O’Hara, Shima \& Zhang 2009). 
Trigonospila Pokorny, 1886

\section{Trigonospila ludio (Zetterstedt, 1849)}

Tachina ludio Zetterstedt, 1849: 3233. Type locality: Denmark.

Distribution: Palaearctic: Europe: Danmark, Poland, Austrian and Italian Alps, Russia (NW. European part, E. Siberia (E. Saian Mts.), S. Far East (Primorsk Territory), Japan (Hokkaido, Honshu, Shikoku, Kyushu) (Herting \& Dely-Draskovits 1993, Richter 2004, Draber-Mońko 2007, Shima 2014), Korea (Byun \& Han 2010), China Provinces (Liaoning, Sichuan, Shaanxi, Shanxi, Tibet); Oriental: China Provinces (Guangxi Zhuangzu A.R., Guizhou, Hunan, Yunnan), India, Myanmar (O’Hara, Shima \& Zhang 2009).

\section{Trigonospila transvittata (Pandellé, 1896)}

Succingulum transvittatum Pandellé, 1896: 148. Type locality: France, Var Hyéres.

Material examined: North Korea, Kesǒng-si Prov., Kesǒng-City, Kesǒng near tomb of King Kongmin, 10. 08. 1987, 1 male, leg. E. Kierych.

Distribution: Palaearctic: Europe: France, Spain (Herting \& Dely-Draskovits 1993), Japan (Honshu, Shikoku, Kyushu) (Shima 2014), Korea (Lee \& Kwon 1981, Byun \& Han 2010, Paeck et al., 2010), China (Sichuan Prov.); Oriental: Taiwan, China Provinces (Fujian, Guangdong, Guangxi Zhuangzu A.R., Guizhou, Hainan, Hunan, Yunnan, Zhejiang), Japan (Ryukyus Islands), Malaysia, Thailand; Australasian: Melanesia (O’Hara, Shima \& Zhang 2009).

Vibrissina Rondani, 1861

\section{Vibrissina debilitata (Pandellé, 1896)}

Latreillia debilitata Pandellé, 1896: 110. Type locality: France, Hautes-Pyrénées, Tarbes.

Distribution: Palaearctic: Europe, Russia (NW. Russia) (Richter 2004), Korea (Shima 1983), China Provinces: Henan, Heilongjiang, Hunan, Jilin, Liaoning, Sichuan, Shaanxi (O’Hara, Shima \& Zhang 2009).

\section{Vibrissina turrita (Meigen, 1824)}

Tachina turrita Meigen, 1824: 401. Type locality: not given ((probably Germany, Kiel and Hamburg).

Distribution: Palaearctic: Europe, Transcaucasia, the Russian S. Far East, Korean Peninsula (Shima 1983, Paek et al. 2010), Japan (Hokkaido, Honshu, Shikoku, Kyushu)(Shima 2014), China (distributet almost all over the country, but has not been recorded from the Provinces: Guangdong, Gansu, Hainan, Hong Kong S.A.R., Hubei, Macao S.A.R., Ningxia Huizu A.R., Qinghai, Xinjiang Uygur A.R.) (O’Hara, Shima \& Zhang 2009).

Zaira Robineau-Desvoidy, 1830

\section{Zaira cinerea (Fallén, 1810)*}

Tachina cinerea Fallén, 1810: 268. Type locality: Sweden, Skåne, Äsperöd (as Esperöd).

Material examined: North Korea, Kangvǒn-do Prov., Munčhǒn, 12 km NW of Vǒnsan, 19. 09. 1970, 1 male, leg. R. Bielawski and M. Mroczkowski.

Distribution: Palaearctica: Europe (all), Russia (W. Russia, W. Siberia, E. Siberia, S. Far East) (Richter 2004), Japan (Hokkaido, Honshu, Shikoku) (Shima 2014), Mongolia, China Provinces (Beijing M., Heilongjiang, Inner Mongolia, Qinghai, Shanxi) (O'Hara, Shima \& Zhang 2009). First record from Korea. 
Tribe Eryciini

Aplomya Robineau-Desvoidy, 1830

\section{Aplomya confinis (Fallén, 1820)}

Tachina confinis Fallén, 1820: 32. Type locality: Sweden, Gotland.

Distribution: Palaearctic: Europe (all), Russia (W. Russia, S. Siberia, E. Siberia, S. Far East (Khabarovsk Territory, Primorsk Territory, South Kuril Island (Kunashir) (Richter 2004), Korea (Lee \& Kwon 1981, Paek et al. 2010), Japan (Hokkaido, Honshu, Shikoku, Kyushu, Tshushima Islands) (Shima 2014), Transcaucasia, Israel, N. Africa, M. Asia, Mongolia, China Provinces (Beijing M., Hebei, Heilongiang, Jilin, Liaoning, Inner Mongolia, Qinghai, Sichuan, Shaanxi, Shanxi, Xinjiang Uygur A.R., Xizang A.R.; Oriental: China Provinces (Guangdong, Hainan, Yunnan); Afrotropical: Yemen (O’Hara, Shima \& Zhang 2009).

Carcelia Robineau-Desvoidy, 1830

\section{Carcelia (Carcelia) gnava (Meigen, 1824)}

Tachina gnava Meigen, 1824: 330. Type locality: Sweden, Skåne, Äsperöd.

Distribution: Palaearctic: Europe (all), Transcaucasia, Russia (W. Russia, E. Siberia, S. Far East, Primorsk Territory, Sakhalin, South Kuril Island (Kunashir)) (Richter 2004), Korea (Kim \& Kim 1972), Japan (Hokkaido, Honshu, Shikoku, Kyushu) (Shima 2014), China Provinces (Beijing M., Hebei, Henan, Heilongiang, Jilin, Liaoning, Sichuan, Shanxi); Oriental: China Provinces ( Fujian, Guangxi Zhuangzu A.R., Guizhou, Hunan, Yunnan, Zhejiang) (O’Hara, Shima \& Zhang 2009).

Carcelina Mesnil, 1944

\section{Carcelina pallidipes (Uéda, 1960)*}

Carcelia pallidipes Uéda, 1960: 112. Type locality: Japan, Hokkaido, Sapporo.

Material examined: North Korea, Kangvǒn-do Prov. Vǒnsan, 4. 09. 1966, 1 male, 1 female, leg. C. Dziadosz and H. Szelęgiewicz.

Distribution: Palaearctic: Japan (Hokkaido, Honshu, Shikoku, Kyushu) (Shima 2014), China Provinces (Beijing M., Heilongiang, Jilin, Liaoning, Sichuan, Shaanxi, Shanxi); Oriental: China Provinces (Fujian, Zhejiang) (O’Hara, Shima \& Zhang 2009). First record from Korea.

Epicampocera Macquart, 1849

\section{Epicampocera succincta (Meigen, 1824)}

Tachina succincta Meigen, 1824: 335. Type locality: not given (probably Germany, Stolberg).

Distribution: Palaearctic: Europe (all), Transcaucasia, Russia (W. Russia, S. Siberia (Tomsk Prov.), S. Far East (Primorsk Territory, South Kuril Island (Kunashir)) (Richter 2004), Korea (Kim \& Kim 1972b), Japan (Hokkaido, Honshu, Shikoku, Kyushu) (Shima 2014), China Provinces (Hebei, Heilongiang, Jilin, Sichuan, Shaanxi); Oriental: China (Hunan Prov.) (O’Hara, Shima \& Zhang 2009). 
Isosturmia Townsend, 1927

\section{Isosturmia picta (Baranov, 1932)}

Sturmia picta Baranov, 1932: 77. Type locality: Taiwan, P'ingtung, Hsien, Changkou (as "Kankau", near Hengch'un).

Distribution: Palaearctic: Korea (Kim 1971, Lee et al. 1981), Japan (Hokkaido, Honshu, Shikoku, Kyushu, Tshushima Island) (Shima 2014), China Provinces (Anhui, Beijing M., Hong Kong S.A.R., Hubei, Jiangsu, Sichuan, Shanghai M., Shanxi); Oriental: Taiwan, China Provinces (Fujian, Guangdong, Guangxi Zhuangzu A.R., Guizhou, Hainan, Hunan, Jiangxi, Yunnan, Zhejiang), India, Indonesia (Jawa), Malaysia (Pen. Malaysia, E. Malaysia), Nepal, Philippines, Sri Lanka, Thailand, ? Vietnam (O’Hara, Shima \& Zhang 2009).

Lydella Robineau-Desvoidy, 1830

\section{Lydella stabulans (Meigen, 1824)}

Tachina stabulans Meigen, 1824: 306. Type locality: not given (Germany, Holstein, according to Herting 1972: 13)

Distribution: Palaearctic: Europe (all), Transcaucasia, C. Asia, Russia (W. Russia, W. Siberia, E. Siberia), China (Sichuan Prov.) (O’Hara, Shima \& Zhang 2009), Korea (Nakayama 1929, Paek et al. 2010).

Prooppia Townsend, 1926

\section{Prooppia agnatella Mesnil, 1955*}

Phebellia (Phebellia) agnatella Mesnil, 1955: 458. Type locality: China, Jiangsu, Suzhou (as Suchow).

Material examined: North Korea, Phjǒngjang-si Prov. Jongak-san Mt., Debong ad Phjǒngjang, 7. 06. 1990, 1 male, leg. E. Chudzicka, E. Kierych and R. Pisarska.

Distribution: Palaearctic: Japan (Hokkaido, Honshu, Kyushu) (Shima 2014), China Provinces (Hebei, Jiangsu, Liaoning, Shanghai, Shanxi); Oriental: China (Yunnan Prov.) (O’Hara, Shima \& Zhang 2009). First record from Korea.

\section{Prooppia latipalpis (Shima, 1981)*}

Phebellia latipalpis Shima, 1981: 63. Type locality: Japan, Hokkaidō, Sorachi, Mt. Yubari.

Material examined: North Korea, Phjǒngjang-si Prov. Phjǒngjang, Tesǒng-san park, 6. 06. 1990, 1 male, leg. E. Chudzicka, E. Kierych and R. Pisarska.

Distribution: Palaearctic: Russia (S. Far East: Primorie Territory) (Richter 2004), Japan (Hokkaido, Honshu, Kyushu) (Shima 2014), China Provinces (Shanghai, Shaanxi) (O’Hara, Shima \& Zhang 2009). First record from Korea.

Pseudoperichaeta Brauer et Bergenstamm, 1889

\section{Pseudoperichaeta nigrolineata (Walker, 1853)}

Tachina nigrolineata Walker, 1853: 85. Type locality: England.

Distribution: Palaearctic: Europe (all), Transcaucasia, Russia (W. Russia, S. Siberia (Altai, Tuva), S. Far East: South Kuril Island (Kunashir)) (Richter 2004), Japan (Hokkaido, Honshu, Shikoku, Kyushu, Tshushima Island) (Shima 2014), Korea (Herting \& Dely-Draskovits 1993), China Provinces (Anhui, Beijing M., Chongqing M., Hebei, Henan, Hubei, Jiangsu, Liaoning, Sichuan, Shandong, Shanghai M., Shaanxi, Shanxi, Xinjiang Uygur A.R.); Oriental: Japan 
(Ryukyu Islands) (Shima 2014), China Provinces (Fujian, Guangdong, Guangxi Zhuangzu A.R., Guizhou, Hunan, Jiangxi, Zhejiang) (O’Hara, Shima \& Zhang 2009).

Senometopia Macquart, 1834

\section{Senometopia excisa (Fallén, 1820)}

Tachina excisa Fallén, 1820: 32. Type locality: Sweden, Östergotland, Lärketorp.

Distribution: Palaearctic: Europe (all), Russia (S. Far East: Primorsk Territory, Khabarovsk Territory, South Kuril Island (Iturup, Kunashir)) (Richter 2004), Korea (Kim 1971, Lee \& Kwon 1981, Paek et al. 2010 as Carcelia excisa (Fall.), Japan (Hokkaido, Honshu, Shikoku, Kyushu, Tshushima Islands) (Shima 2014), China Provinces (Anhui, Beijing M., Chongqing M., Gansu, Hebei, Henan, Hong Kong S.A.R., Heilongjiang, Hubei, Jilin, Jiangsu, Liaoning, Inner Mongolia, Sichuan, Shandong, Shanghai M., Shaanxi, Shanxi, Tianjin, Tibet); Oriental: Japan (Ryukyu Islands) (Shima 2014), Taiwan, China Provinces (Fujian, Guangdong, Guangxi Zhuangzu A.R., Guizhou, Hainnan, Hunan, Jiangxi, Yunnan, Zhejiang), India, Sri Lanka (O’Hara, Shima \& Zhang 2009).

\section{Senometopia orientalis (Shima, 1968)*}

Eucarcelia orientalis (Shima, 1968): 521. Type locality: Japan, Ryukyu Is., Amami-Ō-shima, Yuwandake.

Material examined: North Korea, Phjǒngan-namdo Prov., Sunan distr., Sǒkam-Chǒsudži, 2.09.1970, 1 female, leg. R. Bielawski and M. Mroczkowski.

Distribution: Palaearctic: China Provinces (Beijing M., Sichuan, Shanxi); Oriental: Japan (Ryuku Is.), China Provinces (Fujian, Guangxi Zhuangzu A.R., Guizhou, Jiangsu, Jiangsi, Yunnan, Zhejiang) (O’Hara, Shima \& Zhang 2009). First record from Korea.

\section{Senometopia separata (Rondani, 1859)*}

Exorista separata (Rondani, 1859): 134. Type locality: Italy, Parma.

Material examined: North Korea, Kangvǒn-do Prov., Kymgan-san Mts., Onjong-ri near Kymgan-san hotel, 23-25.06. 1990, 2 females, leg. E. Chudzicka, E. Kierych and R. Pisarska.

Distribution: Palaearctic: Europe northwards to N. German, N. Poland, S. Sweden, Ukraine, Russia (S. Siberia: Krasnoyarsk, Novosibirsk; S. Far East: Sakhalin) (Richter 2004), Japan (Hokkaido, Honshu, Kyushu (Shima 2014), China Provinces (Hebei, Heilongjiang, Hubei, Jiangsu, Liaoning, Sichuan); Oriental: China Provinces (Fujian, Guangdong, Hainan, Hunan, Yunnan, Zhejiang) (O’Hara, Shima \& Zhang 2009). First record from Korea.

\section{Thecocarcelia Townsend, 1933}

\section{Thecocarcelia sumatrana Baranov, 1932}

Sturmia sumatrana Baranov, 1932: 1. Type locality: Indonesia, Sumatera, Medan.

Thelycarcelia trix Townsend, 1933: 475. Type locality: Taiwan, P'ingtung Hsien, Changkou [as "Kangkau”, near Hengch"un].

Distribution: Palaearctic: Korea (Shima 1968, O’Hara, Shima \& Zhang 2009), Japan (Hokkaido, Honshu, Shikoku, Kyushu, Tsushima Island) (Shima 2014), China Provinces (Heilongjiang, Hubei, Jilin); Oriental: Taiwan. China Provinces (Fujian, Guangdong, Guangxi Zhuangzu A.R., Hainan, Hunan, Jiangxi, Yunnan, Zhejiang), India, Indonesia (Sumatera), Malaysia (Pen. Malaysia, E. Malaysia), Philippines, Sri Lanka, Thailand, Vietnam (O'Hara, Shima \& Zhang 2009). 
Thelyconychia Brauer et Bergenstamm, 1889

\section{Thelyconychia solivaga (Rondani, 1861)*}

Masicera (Ceromasia) solivaga Rondani, 1861: 24. Type locality: Italy, Parma.

Material examined: North Korea, Hamhyng-si Prov., Hyngnam, 11.06. 1965, 1 female, leg. M. Mroczkowski and A. Riedel; Čojang, 15 km SW ad Hamhyng, 25.09.1970, 1 female, leg. R. Bielawski and M. Mroczkowski.

Distribution: Palaearctic: Europe (W. Europe, C. Europe, E. Europe, S. Europe), Transcaucasia, M. East, N. Africa, C. Asia, Russia: E. Siberia, S. Far East (S. Primorsk Territory) (Richter 2004, Draber-Mońko 2007), Japan (Hokkaido), China (NE China); Oriental (Pakistan), Afrotropical: Botswana, Yemen (O’Hara, Shima \& Zhang 2009). First record from Korea.

\section{Tribe Exoristini}

Chaetexorista Brauer et Bergenstamm, 1894

\section{Chaetexorista pavlovskyi (Stackelberg, 1943)*}

Carcelia (Megacarcelia) pavlovskyi Stackelberg, 1943: 163. Type locality: Russia, Primorskiy Kray, upper Komarovka (as "Suputinka") river.

Material examined: North Korea, Phjǒngan-pukto Prov, Tephun ad Kujang-dong, Mjohjang-san Mts., 4.09.1959, 2 females, leg. B. Pisarski. Kangvǒn-do Prov., Munčhǒn 12 km NW ad Vǒnsan, 19.09.1970, 1 female, leg. R. Bielawski and M. Mroczkowski.

Distribution: Palaearctic: Russia (S. Far East: Primorsk Territory, Sakhalin) (Richter 2004, Shima 2014), Japan (Honshu) (Shima 2014), China (O’Hara, Shima \& Zhang 2009); Oriental: Taiwan (Shima 2014). First record from Korea.

\section{Exorista Meigen, 1803}

Adenia Robineau-Desvoidy, 1863

\section{Exorista (Adenia) rustica (Fallén, 1810)}

Tachina rustica Fallén, 1810: 264. Type locality: Sweden, Skåne, Äsperöd (as Esperöd').

Material examined: North Korea, N. Hamgyong Prov., Chǒngdžin-City, Desso-ri, 15. 06. 1990, 1 female, leg. E. Chudzicka, E. Kierych and R. Pisarska.

Distribution: Palaearctic: Europe, Transcaucasia, Turkey, Israel, Mongolia, Russia (W. Russia, W. Siberia, S. Siberia, E. Siberia, the Russian Far East (Amuria, Khabarovsk Territory)) (Herting \& Dely-Draskovits 1993, Richter 2004), Korea (Kim \& Kim 1971, Chao \& Xue 1998), China Provinces (Anhui, Beijing M., Hebei, Heilongjiang, Jilin, Jangsu, Liaoning, Inner Mongolia, Qinghai, Sichuan, Shandong, Shanghai M., Shanxi, Tianjin M., Xinjiang Uygur A.R., Xizang A.R.); Oriental: Taiwan, China Provinces (Fujian, Jiangxi, Yunnan, Zhejlang) (O’Hara, Shima \& Zhang 2009).

\section{Exorista Meigen, 1803}

\section{Exorista (Exorista) japonica (Townsend, 1909)}

Tachina japonica Townsend, 1909: 247. Type locality: Japan, Honshū, Tokyo vicinity.

Material examined: North Korea, Phjǒngjang-si Prov., Phjǒngjang, Moran-bong, 27.08.1970, 1 male, leg. R. Bielawski and M. Mroczkowski. 
Distribution: Palaearctic: Korea (Paek et al. 2010), Japan (Hokkaido, Honshu, Shikoku, Kyushu, Tsushima Island) (Shima 2014), China (distributed almost all over the country, but has not been recorded from the Provinces: Macao S.A.R., Qinghai, Shaanxi); Oriental: Japan (Ryukyu Islands) (Shima 2014), Taiwan, India Indonesia, Malaysia, Nepal, Philippines, Thailand, Vietnam (O’Hara, Shima \& Zhang 2009).

\section{Exorista (Eorista) larvarum (Linnaeus, 1758)*}

Musca larvarum Linnaeus, 1758: 596. Type locality: not given (Europe).

Material examined: North Korea, Phjǒngjang-si Prov., Jongsǒng distr., Maram ad Phjǒngjang, 30. 07.1959, 1 male, leg. B. Pisarski and J Prószyński. Chǒngdžin-si Prov., Chǒngdžin, 12.09.1966, 1 male, leg. C. Dziadosz and H. Szelęgiewicz,

Distribution: Palaearctic: Europe (all), Transcaucasia, M. East, N. Africa, C. Asia, Mongolia, Russia (W. Russia, W. Siberia, E. Siberia, S. Far East: Primorsk Territory, S. Kuril Islands: Kunashir, Shikotan) (Richter 2004, O'Hara, Shima \& Zhang 2009). Japan (Hokkaido, Honshu, Kyushu) (Shima 2014), China Provinces (Anhui, Beijing M., Gansu, Hebei, Henen, Heilongjiang, Jilin, Jiangsu, Liaoning, Inner Mongolia, Ningxia Huizu A.R., Quinghai, Sichuan, Shandong, Shanghai M., Shaanxi, Shanxi, Tianjin M., Xinjiang Uygur A.R.); Oriental: Taiwan, China Provinces (Fujian, Guangdong, Guangxi Zhuangzu A.R., Guizhou, Hainan, Hunan, Jiangxi, Zhejiang), India; Nearctic: Yukon, introduced and established in Ontario, Québec, and New England (O’Hara, Shima \& Zhang 2009). First record from Korea.

Podotachina Brauer et Bergenstamm, 1891

\section{Exorista (Podotachina) sorbilans (Wiedemann, 1830)}

Tachina sorbilans Wiedemann, 1830: 311. Type locality: Canary Islands, Tenerife.

Distribution: Palaearctic: Europe (W. Europe, E. Europe, S. Europe), C. Asia, Mongolia, N. Africa, China Provinces (Anhui, Beijing M., Chongqing M., Hebei, Henan, Heilongjiang, Hubei, Jilin, Jiangsu, Liaoning, Sichuan, Shandong, Shanghai, Shanxi) (O'Hara, Shima \& Zhang 2009). Korea (Paek et al. 2010), Japan (Hokkaido, Honshu, Shikoku, Kyushu) (Shima 2014); Oriental: Japan (Ryukyu Is.), Taiwan, China Provinces (Fujian, Guangdong, Guangxi Zhuangzu A.R., Guizhou, Hainan, Hunan, Jiangxi, Yunnan, Zhejiang), India, Indonesia, Nepal, Philippines, Sri Lanka, Thailand, Vietnam; Australasian: Papua N.G.; Afrotropical: Cameroon, Kenya, Malawi, Sierra Leone, Uganda (O’Hara, Shima \& Zhang 2009).

Spixomyia Crosskey, 1967

\section{Exorista (Spixomyia) bisetosa Mesnil, 1940}

Exorista (Scotiella) bisetosa Mesnil, 1940: 39. Type locality: China, near Shanghai, Xujiahui (as Zi ka wei).

Distribution: Palaearctic: Korea (Han et al. 2004, Paek et al. 2010), Japan (Honshu, Shikoku, Kyushu, Tsushima Island) (Shima 2014), China Provinces (Anhui, Beijing M., Hebei, Hong Kong S.A.R., Jilin, Jiangsu, Inner Mongolia, Shandong, Shanghai M., Shanxi, Tianjin M., Tibet); Oriental: Japan (Ryukyu Is.), Taiwan, China Provinces (Fujian, Guangdong, Guangxi Zhuangzu A.R., Hainan, Hunan, Jiangxi, Zhejiang), Indonesia (Jawa); Australasian: ? Melanesia (O’Hara, Shima \& Zhang 2009). 


\section{Exorista (Spixomyia) quadriseta (Baranov, 1932)}

Eutachina quadriseta Baranov, 1932: 91. Type locality: Taiwan, Kaohsiung Hsien, Chiahsien Hsiang (as "Sokutsu").

Distribution:Palaearctic: Korea (Chao \& Xue 1998), China Provinces (Jiangsu, Sichuan, Shaanxi); Oriental: Taiwan, China Provinces (Hunan, Yunnan, Zhejiang); Australasian: Australia, Melanesia, Papua N.G. (O’Hara, Shima \& Zhang 2009).

Parasetigena Brauer et Bergenstamm, 1891

\section{Parasetigena silvestris (Robineau-Desvoidy, 1863)}

Duponchelia silvestris Robineau-Desvoidy, 1863: 531. Type locality: not given (France, probably near Paris).

Material examined: North Korea, Phjǒngan-pukto Prov., Mjohjang-san Mts., at the foot of Hyangro Peck, 9-12. 06. 1990, 1 male, leg. E. Chudzicka, E. Kierych and R. Pisarska.

Distribution: Palaearctica: Europa (all), Transcaucasia, Russia (E.Siberia: Chita, Yakutia, S. Far East: Primorsk Territory, Khabarovsk Territory) (Richter 2004), Korea (Pemberton et al. 1993), Japan (Hokkaido, Honshu, Shikoku, Kyushu, Tsushima Island) (Shima 2014), China Provinces (Heilongjiang, Jilin, Liaoning) (O’Hara, Shima \& Zhang 2009).

\section{Phorinia Robineau-Desvoidy, 1830}

\section{Phorinia breviata Tachi and Shima, 2006*}

Phorinia breviata Tachi and Shima, 2006: 260.Type locality: Japan, Fukuoka Pref., Fukuoka City, Mt. Aburayama.

Material examined: North Korea, Kangvǒn-do Prov., Kumgang-san Mts., Onjong-ri near Kymgan-san hotel, 28. 08. 1987, 1 male, leg. E. Kierych.

Distribution: Palaearctic: Japan (Hokkaido, Honshu, Shikoku, Tsushima Island). Oriental and Oceanian regions (Tachi and Shima 2006). First record from Korea.

Remarks: In the first version of this paper I have given the information that among the specimens examined I found male belonging to the Phorinia aurifrons Robineau-Desvoidy, 1830 (after the Key to the insects of Russian Far East. Vol. VI. Diptera and Siphonaptera. Pt 3. Vladivostok. 2004. 124. Fam. Tachinidae. Richter: 197-198). However, one of reviewers, in his comments wrote: "Phorinia aurifrons Robineau-Desvoidy (...) is considered to be misidentified from East Asia by some authors (see Tachi \& Shima 2006, O'Hara et al. 2009, Shima 2014). The author is recommended to confirm identification of this species. If the species $P$. aurifrons really occurs in North Korea, it is very interesting".

My repeated examination of the specimen from Korea confirmed the suspicions of reviewer. Finally, I decided that it was $P$. breviata Tachi and Shima.

Phorocera Robineau-Desvoidy, 1830

\section{Phorocera assimilis (Fallén, 1810)*}

Tachina assimilis Fallén, 1810: 283. Type locality: Sweden.

Material examined: North Korea, Phjǒngan-pukto Prov., Mjohjang-san Mts., at the foot of Hyangro Peck, 9-12. 06. 1990, 1 male, leg. E. Chudzicka, E. Kierych and R. Pisarska.

Distribution: Palaearctic: Europe (all), Transcaucasia, C. Asia, Russia (W. Siberia, SE. Siberia, S. Far East: Primorsk Territory, Khabarovsk Territory, S. Kuril Islands (Kunashir)), 
Japan (Hokkaido, Honshu, Shikoku, Kyushu) (Shima 2014), China Provinces (Heilongjiang, Liaoning, Shanxi) (Richter 2004, O’Hara, Shima \& Zhang 2009). First record from Korea.

Tribe Goniini

Allophorocera Hendel, 1901

\section{Allophorocera rutila (Meigen. 1824)}

Tachina rutila Meigen. 1824: 382. Type locality: Italy, Torino.

Distribution: Palaearctic: Europe (W. Europe, S. Europe), Transcaucasia, The Russian Far East (Amur Prov., Primorsk Territory, S, Kuril Islands (Kunashir)) (Richter 2004), Korea (Kim et al. 1971. Lee et Kwon 1981, Paek et al. 2010), Japan (Hokkaido, Honshu), China Provinces (Beijing M., Liaoning) (O’Hara, Shima \& Zhang 2009, Shima 2014).

Erythrocera Robineau-Desvoidy, 1848

\section{Erythrocera nigripes (Robineau-Desvoidy, 1830)*}

Phryno nigripes Robineau-Desvoidy, 1830: 144. Type locality: France, St.-Sauveur.

Material examined: North Korea, N Hamgyong Prov., Chongdžin-City, Kyowon-ri, 16. 06. 1990, 1 male, leg. E. Chudzicka, E. Kierych and R. Pisarska.

Distribution: Palaearctic: W.Europe northwards to France, Germany and Poland, Ukraine, Moldova, Russia (European part of Russia, S. Far East (Primorsk Territory) (Richter 2004, Draber-Mońko 2007). First record from Korea.

Blepharipa Rondani, 1856

\section{Blepharipa schineri (Mesnil, 1939)}

Blepharipoda schineri Mesnil, 1939: 32. Type locality: not given (Austria).

Distribution: Palaearctic: Europe (British Is., W. Europe. C. Europe, E. Europe. S. Europe), Russia (European part of Russia, S. Siberia (Krasnoyarsk, Tomsk Prov., Novosibirsk Prov.), S. Far East (Primorsk Territory, Khabarovsk Territory, S, Kuril Islands: Kunashir (Richter 2004, Draber-Mońko 2007), Korea (Pemberton et al. 1993), Japan (Hokkaido, Honshu, Kyushu, Tsushima Is.) (Shima 2004), China Provinces (Heilongjiang, Hubei, Jilin, Jiangsu, Liaoning, Inner Mongolia, Sichuan, Shaanxi); Oriental: China Provinces (Guizhou, Hunan, Zhejiang) (O’Hara, Shima \& Zhang 2009).

\section{Blepharipa zebina (Walker, 1849)}

Tachina zebina Walker, 1849: 772. Type locality: India, North Bengal.

Material examined: North Korea, Hamgjǒng-pukto Prov., Kjǒngsǒng distr., near Kjǒngsǒng hill, 3. 09. 1987, 1 female, leg. J. Sawoniewicz.

Distribution: Palaearctic: Russia (S. Far East: Primorsk Territory) (Richter 2004), Korea (Shima 2006, Paek et al. 2010), Japan (Hokkaido, Honshu, Kyushu, Tsushima Is.) (Shima 2014), China (distributed almost all over the country, but has not been recorded from the Provinces: Hong Kong S.A.R., Macao S.A.R., Qinghai, Xinjiang Uygur A.R.); Oriental: Japan (Ryukyu Is) (Shima 2014), Taiwan, India, Myanmar, Nepal, Sri Lanka, Thailand (O’Hara, Shima \& Zhang 2009). 
Dolichocolon Brauer et Bergenstamm, 1889

\section{Dolichocolon abstrusum Cerretti et Shima, 2011}

Dolichocolon abstrusum Cerretti et Shima, 2011: 568. Type locality: Japan, Honshu, Pref. Saitama, Yorii.

Distribution: Palaearctic: Koraea (Cerretti \& Shima 2011), Japan (Honshu) (Cerretti \& Shima 2011, Shima 2014).

Frontina Meigen, 1838

\section{Frontina femorata Shima, 1988}

Frontina femorata Shima, 1988: 33. Type locality: Japan, Hokkaido, Mt. Rausu (200-800 m).

Distribution: Palaearctic: Korean Peninsula (Kim 1981, Lee et Han 2008, Paek et al. 2010, Shima 2014), Japan (Hokkaido, Honshu) (Shima 2014), China: Jilin Prov. (O’Hara, Shima \& Zhang 2009).

\section{Frontina laeta (Meigen, 1824)}

Tachina laeta Meigen, 1824: 381. Type locality: not given (Europe).

Distribution: Palaearctic: Europe (all), Transcaucasia, Kazakhstan, Russia (W. Russia, W. Siberia, E. Siberia, S. Far East (Amur Prov., Khabarovsk Territory, Primorsk Territory) (Richter 2004), Korea (Kim 1981, Lee et Han 2008, Paek 2010), Japan: Hokkaido, Honshu (Shima 2004), China Provinces (Henan, Jilin, Jiangsu, Inner Mongolia, Shandong); Oriental: China (Zhejiang Prov.) (O’Hara, Shima \& Zhang 2009).

\section{Frontina tricolor Shima, 1988}

Frontina tricolor Shima, 1988: 33. Type locality: Japan, Honshu, Pref. Saitama, Mt. Buko.

Distribution: Palaearctic: Korean Peninsula (Lee et Han 2008, Paek et al. 2010, Shima 2014), Japan (Honshu, Kyushu) (Shima 2014).

Gonia Meigen, 1803

\section{Gonia chinensis Wiedemann, 1824}

Gonia chinensis Wiedemann, 1824: 47. Type locality: China.

Distribution: Palaearctic: C. Asia (Herting \& Dely-Draskovits 1993), Korean Peninsula (Doi 1938, Paek et al. 2010), Japan (Hokkaido, Honshu, Shikoku, Kyushu) (Shima 2014), China Provinces (Anhui, Beijing M., Chongqing M., Gansu, Hebei, Henan, Hong Kong S.A.R., Heilongjiang, Hubei, Jiangsu, Inner Mongolia, Sichuan, Shandong, Shanghai M., Shaanxi, Shanxi, Tianjin M., Tibet); Oriental: Taiwan, China Provinces (Fujian, Guangdong, Guangxi Zhuanangzu A.R., Guizhu, Hainan, Hunan, Jiangxi, Yunnan, Zhejiang), India, Malaysia. (? L. Sunda Is.), Nepal, Pakistan, Philippines, Vietnam (O’Hara, Shima \& Zhang 2009).

\section{Gonia distinguenda Herting, 1963}

Gonia distinguenda Herting, 1963: 106. Type locality: Switzerland, Wallis, Grengiols.

Distribution: Palaearctic: Europe (Switzerland, Hungary, Italy, Germany) (Herting \& DelyDraskovits 1993), Korea (Lee et Han 2010). 


\section{Gonia divisa Meigen, 1826}

Gonia divisa Meigen, 1826: 4. Type locality: Austria.

Distribution: Palaearctic: Europe northwards to S. Sweden and northern part of Russia; Asia: Russia: S. Siberia (Krasnoyarsk, Tuva, Tomsk Prov., Novosibirsk Prov.), S. Far East (Khabarovsk Territory, Primorsk Territory) (Richter 2004), Korea (Lee et Han 2010), China (Beijing M. Prov.) (O’Hara, Shima \& Zhang 2009), Japan (Hokkaido, Honshu) (Shima 2014).

\section{Gonia foersteri Meigen. 1838}

Gonia foersteri Meigen. 1838: 246. Type locality: Germany, Stolberg.

Distribution: Palaearctic: Europe (Austria, Germany, Poland, Czechoslovakia, Greece, Italy, Russia (S. European part), Asia (Israel) (Herting \& Dely-Draskovits 1993, DraberMońko 2007), Korea (Kim 1971, Lee et Han 2010).

\section{Gonia klapperichi (Mesnil, 1956)}

Turanogonia klapperichi Mesnil, 1956: 532. Type locality: China, Fujian, Kwangtsch.

Distribution: Palaearctic: Korea (Han et al. 2004, Paek et al. 2010), China Provinces (Liaoning, Qinghai, Sichuan, Shaanxi, Xinjiang Uygur A.R.); Oriental: China Provinces (Fujian, Guangdong, Guangxi Zhuangzu A.R., Guizhou, Yunnan, Zhejiang), India, Myanmar (O’Hara, Shima \& Zhang 2009).

\section{Gonia nigricoma Lee et Han 2010}

Gonia nigricoma Lee et Han 2010: 188. Type locality: South Korea.

Distribution: Palaearctic: Palaearctic: Korea (Lee et Han 2010).

\section{Gonia olgae (Rohdendorf, 1927)}

Salmacia olgae Rohdendorf, 1927: 94. Type locality: Uzbekistan, Tashkent.

Distribution: Palaearctic: Europe: Spain; Asia: Turkey, C. Asia (Herting \& Dely-Draskovits 1993), Korea (Lee et Han 2010).

\section{Gonia picea (Robineau-Desvoidy, 1830)*}

Spallanzania picea Robineau-Desvoidy, 1830: 78. Type localities: France and Spain.

Material examined: North Korea, Phjǒngjang Prov., Moran-yong, 27. 08. 1970, 1 male, leg. R. Bielawski and M. Mroczkowski.

Distribution: Palaearctic: Europe: Spain, France, Hungary, Poland, Germany, Great Britain, Sweden, Russia (European part), Transcaucasia, Israel (Herting \& Dely-Draskovits 1993, Draber-Mońko 2007), Turkmenia, Russia (S. Siberia (Irkutsk), N. Far East: Kamchatka) (Richter 2004), Japan (Hokkaido, Honshu, Shikoku, Kyushu) (Shima 2014). China Provinces (Anhui, Beijing M., Hebei, Henan, Heilongjiang, Jilin, Jiangsu, Liaoning, Inner Mongolia, Qinghai, Sichuan, Shandong, Shanghai M., Shaanxi, Shanxi, Tianjin M., Xinjiang Uygur A. R., Tibet); Oriental: Taiwan, China Provinces (Fujian, Guizhou, Hunan, Jiangxi, Yunnan, Zhejiang) (O’Hara, Shima \& Zhang 2009). First record from Korea. 


\section{Gonia ussuriensis (Rohdendorf, 1928}

Salmacia (Chrysocerogonia) ussuriensis Rohdendorf, 1927: 94. Type localities: Russia, Primorskij Kray, Yakovlevka (as "Jakovlevka"), and Steklyannaya (as"Stekljanucha", about 9 km ENE of Shkotovo).

Distribution: Palaearctic: Russia (S. Far East: Primorsk Territory) (Richter 2004), Korean Pennisula (Lee \& Han 2010), Japan (Honshu, Shikoku, Kyushu) (Shima 2014), China Provinces (Heilongjiang, Shanghai M.) (O’Hara, Shima \& Zhang 2009).

Platymya Robineau-Desvoidy, 1830

\section{Platymya fimbriata (Meigen, 1824)}

Tachina fimbriata Meigen, 1824: 337. Type locality: not given (probably Germany, Stolberg).

Distribution: Palaearctic: Europa (all), Transcaucasia, C. Asia, M. East, Mongolia, Russia (W. Russia, S. Siberia, the Russian Far East: Kamchatka, Primorsk Territory, Sakhalin, S. Kuril Islands (Iturup, Kunashir, Shikotan)) (Richter 2004), Korea (Chao et Xue 1998), China Provinces (Chongqing M., Heilongjiang, Sichuan, Shanxi, Tibet); Oriental: China Provinces (Guizhou, Yunnan) (O’Hara, Shima \& Zhang 2009).

Pseudogonia Brauer et Bergenstamm, 1889

\section{Pseudogonia rufifrons (Wiedemann, 1830)}

Tachina rufifrons Wiedemann, 1830: 318. Type locality: China.

Distribution: Palaearctic: Europe (W. Europe, C. Europe, S. Europe, E. Europe), Transcaucasia, M. East, N. Africa, Kazakhstan, C. Asia, Mongolia, Russia (W. Russia, S. Siberia, Far East: Primorsk Territory) (Richter 2004), China Provinces (Anhui, Beijing M., Hebei, Henan, Hong Kong S.A.R., Hubei, Jilin, Jiangsu, Liaoning, Inner Mongolia, Ningxia Huizu A.R., Sichuan, Shandong, Shanghai M., Shanxi, Xinjiang Uygur A. R.) (O'Hara, Shima \& Zhang 2009), Korea (Lee et Han 2007, Paek et al. 2010), Japan (Hokkaido, Honshu, Shikoku, Kyushu) (Shima 2014); Oriental: Japan (Ryukyu Islands) (Shima 2014), Taiwan, China Provinces (Fujian, Guangdong, Guangxi Zhuangzu A.R., Hainan, Jiangxi, Yunnan, Zhejiang), India, Indonesia (Jawa, Sumatra), Malaysia (Pen. Malaysia), Myanmar, Pakistan, Philippines, Thailand; Australasian: Australia, Hawaii, Indonesia (Maluku Is.), Melanesia, Papua N.G.; Afrotropical: widespread, including Cape Verde Islands, Yemen (O’Hara, Shima \& Zhang 2009).

Sturmia Robineau-Desvoidy, 1830

\section{Sturmia bella (Meigen, 1824)}

Tachina bella Meigen, 1824: 317. Type locality: not given (probably Germany, Stolberg).

Distribution: Palaearctic: Europe (all), Transcaucasia, M. East, C. Asia (O’Hara, Shima \& Zhang 2009), Russia (W. Russia, S. Siberia: Altai) (Richter 2004), China Provinces (Gansu, Sichuan) (O’Hara, Shima \& Zhang 2009), Korea (Kim et Nam 1978), Japan (Hokkaido, Honshu, Shikoku, Kyushu) (Shima 2014); Oriental: Japan (Ryukyu Islands) (Shima 2014), ?Taiwan, China Provinces (Fujian, Guangdong, Guangxi Zhuangzu A.R., Hainan, Hunan, Yunnan, Zhejiang), Nepal, Thailand; Australasian: ?Bismarck Arch., Melanesia (O'Hara, Shima \& Zhang 2009). 
Zenillia Robineau-Desvoidy, 1830

\section{Zenillia phrynoides (Baranov, 1939)}

Exorista phrynoides Baranov, 1939: 110. Type locality: Japan, Hokkaidō, Sapporo.

Distribution: Palaearctic: Korean Peninsula (Hirashima 1989, Paek et al. 2010), Japan (Hokkaido, Honshu, Shikoku, Kyushu) (Shima 2014); Oriental: China (Hunan Prov.) (O’Hara, Shima \& Zhang 2009).

Tribe Winthemiini

Nemorilla Rondani, 1856

\section{Nemorilla floralis (Fallén, 1810) *}

Tachina floralis Fallén, 1810: 287. Type locality: Sweden.

Material examined: Hamgjǒng-pukto Prov., Kjǒngsǒng distr., Džuyr ad Čhǒngdžin, 24. 08. 1959, 1 male, leg. B. Pisarski and J. Prószyński.

Distribution: Palaearctic: Europe northwards to Sweden (Herting \& Dely-Draskovits 1993), Russia (S. Far East: Primorsk Territory, Sakhalin, S. Kuril Islands: Iturup, Kunashir) (Richter 2004), Japan (Hokkaido, Honshu, Shikoku, Kyushu, Tsushima Island) (Shima 2014). First record from Korea.

\section{Nemorilla maculosa (Meigen, 1824)}

Tachina maculosa Meigen, 1824: 265. Type localities: Southern France, and Germany, Hamburg, and probably Stolberg.

Distribution: Palaearctic: Europe (Scandinavia, W. Europe, C. Europe, E. Europe, S. Europe), Transcaucasia, M. East, C. Asia, Mongolia, Russia (W. Russia, W. Siberia, S. Far East (Primorsk Territory) (O’Hara, Shima \& Zhang 2009), Korea (Doi 1938, Paek et al. 2010), Japan (Hokkaido, Kyushu) (Shima 2014), China Provinces (Anhui, Beijing M., Hebei, Hong Kong S.A.R., Heilongjiang, Hubei, Jilin, Jiangsu, Liaoning, Inner Mongolia, Sichuan, Shandong, Shanghai M., Shanxi, Tianjin M., Xinjiang Uygur A.R.), N. Africa; Oriental: Japan (Ryuku Islands), Taiwan, China Provinces (Fujian, Guangdong, Guanxi Zhuangzu A.R., Hainan, Hunan, Jiangxi, Zhejiang), India, Myanmar (O’Hara, Shima \& Zhang 2009).

Smidtia Robineau-Desvoidy, 1830

\section{Smidtia gemina (Mesnil, 1949)}

Nemosturmia gemina Mesnil, 1949: 75. Type locality: China, Guling (as Kuling).

Distribution: Palaearctic: Russia (S. Far East: (Primorsk Territory) (Richter 2004), Korean Peninsula (Shima 1996, O’Hara, Shima \& Zhang 2009), Japan (Honshu, Kyushu, Tsushima Island) (Shima 2014), China (Jiangxi Prov.) (O’Hara, Shima \& Zhang 2009).

Winthemia Robineau-Desvoidy, 1830

\section{Winthemia cruentata (Rondani, 1859)}

Chaetolyga cruentata Rondani, 1859: 106. Type locality: Italy, Parma.

Distribution: Palaearctic: Europe (all), Transcaucasia, Russia (W. Russia, E. Siberia (Chita), S. Far East (Khabarovsk Territory)) (Richter 2004), Korea (Han et al. 2004, Paek et al. 
2010), Japan (Hokkaido, Honshu, Kyushu) (Shima 2014), China Provinces (Beijing M., Jilin, Inner Mongolia, Sichuan, Shanxi) (O’Hara, Shima \& Zhang 2009).

\section{Winthemia venusta (Meigen, 1824)}

Tachina venusta Meigen, 1824: 327. Type locality: not given (probably Germany, Stolberg).

Distribution: Palaearctic: Europe (W. Europe, C. Europe, E. Europe, S. Europe), Transcaucasia, Russia (W. Russia, E. Siberia (Chita, Jakutsk), S. Far East (Primorsk Territory, S. Kuril Islands: Kunashir)) (Richter 2004), Japan (Hokkaido, Honshu, Shikoku, Kyushu) (Shima 2014), Korea (Fan 1992, Liu et al. 1998), China Provinces (Beijing M., Gansu, Hebei, Heilongjiang, Jilin, Jiangsu, Liaoning, Inner Mongolia, Sichuan, Shandong, Shanghai M., Shaanxi, Shanxi, Xinjiang Uygur A.R., Tibet); Oriental: Taiwan, China Provinces (Fujian, Guizhou, Hainan, Hunan, Yunnan, Zhejiang) (O’Hara, Shima \& Zhang 2009).

\section{GENERAL REMARKS}

Twenty four species representing 20 genera of the subfamily Exoristinae have been identified in the material in the Museum and Institute of Zoology PAS collection. The species are: Compsilura concinnata (Meig.), Compsiluroides flavipalpis Mesn.*, Medina separata (Meig.)*, Meigenia tridentata Mesn.*, Oswaldia muscaria (Fall.)*, Trigonospila transvittata (Pand.), Zaira cinerea (Fall.)*, Carcelina pallidipes (Ueda)*, Proopia agnatella Mesn.*, P. latipalpis (Shima)*, Senometopia orientalis (Shima)*, S. separata (Rond.)*, Thelyconychia solivaga (Rond.)*, Chaetexorista pavlovskyi (Stack.)*, Exorista (Adenia) rustica (Fall.), E. (Exorista) japonica (Towns.), E. (E.) larvarum (Linn.)*, Parasetigena silvestris (R.-D.), Phorinia breviata Tachi \& Shima*, Phorocera assimilis (Fall.)*, Erythrocera nigripes (R,D.)*, Blepharipa zebina (Walk.), Gonia picaea R.-D.)* and Nemorilla floralis (Fall.)*. Eighteen species (marked with an asterisks) are recorded in Korea for the first time.

The current list of Exoristinae from Korea includes 48 species mentioned earlier by other authors.Nowadays, 72 species belonging to 43 genera of Exoristinae flies are known from the Korean Peninsula.

\section{ACKNOWLEDGEMENTS}

I wish to thank very much the two anonymous reviewers for helpful comments and remarks.

\section{REFERENCES}

ALDRICH J. M.1928. Five new parasitic flies reared from beetles in China and India. Procedings of the United States National Museum 74 (8): 1-7.

BAŃKOWSKA R. \& STERZYŃSKA M. 1997. Faunistic investigations conducted in North Korea by researchers from the Institute of Zoology Polish Academy of Sciences from 1959 to 1990. Fragmenta Faunistica 40: 247-253.

ByUN H. W. 2010. A systematic study of the tribe Blondeliini (Diptera: Tachinidae) in Korea. Ph. D. Dissertation, Yonsei University, Korea, $271 \mathrm{pp.}$

ByUn H. W. \& HAN H. Y. 2009. A taxonomic revision of the genus Metadrinomyia Shima (Diptera: Tachinidae) with descriptions of two new species. Zootaxa 2311: 55-65.

Byun H. W. \& HAN H. Y. 2010. Taxonomic review of the genus Trigonospila Pokorny (Diptera: Tachinidae: Blondeliini) in Korea. The Korean Journal of Systematic Zoology 26 (3): 243-249.

ByUN H. W.\& HAn H. Y. 2011. Lixophaga Townsend (Diptera: Tachinidae: Blondeliini) a newly recorded parasitoid genus in Korea. Journal of Asia-Pacific Entomology 14: 58-62.

CERRETTI P. \& ShIMA H. 2011. World revision of Dolichocolon Brauer and Bergenstamm (Diptera: Tachinidae: Exoristinae: Goniini). Zoological Journal of the Linnean Society 162: 544-584.

Chao C. M. 1998. Tachinidae. Vol. 2. In: Xue W. P. \& Chao C. M. (eds), Flies of China. Vol. 2, pp. $1661-2206$. Liaoning Science and Technology Press, Shenyang, 2425 pp. 
Crosskey R. W. 1977. Family Tachinidae. In: Delfinado M.D. and Hardy D.E. (eds), A Catalog of the Diptera of the Oriental Region.Volume III. Suborder Cyclorrhapha (excluding Division Aschiza), pp. 586-697. The University Press of Hawaii. Honolulu, 854 pp.

DoI H. 1938. A list of the Diptera of Korea. Bulletin de Science Museum Keizyo, Korea. 72 (6): 5-18. [In Japanese].

Draber-MoŃKo A. 2007. Rączyce (Tachinidae). In: Bogdanowicz W., ChUdZiCKA E., PILIPIUK I. \& SKIBIŃSKA E. (eds), Fauna of Poland - characteriristics and checklist of species. Vol.2, pp. 155-160, 233-240. Museum and Instytut of Zoology PAS, Warszawa, $505 \mathrm{pp}$.

DRABER-MoŃKO A. 2008. State of knowledge of the tachinid fauna of Eastern Asia, with new data from North Korea. Part I. Phasiinae. Fragmenta Faunistica 51: 119-137.

DRABER-MoŃKO A. 2011. State of knowledge of the tachinid fauna of Eastern Asia, with new data from North Korea. Part II. Tachiniinae. Fragmenta Faunistica 54: 157-177.

DRABER-MoŃKO A. 2012. State of knowledge of the tachinid fauna of Eastern Asia, with new data from North Korea. Part III. Phasiinae. Supplement. Fragmenta Faunistica 55: 147-153.

DRABER-MoŃKO A. 2015. State of knowledge of the tachinid fauna of Eastern Asia, with new data from North Korea. Part IV. Dexiinae. Fragmenta Faunistica 58: 43-55.

FAN T. 1992. Key to the common flies of China. Beijing Science Press, xiviii+ 992 pp.

Guimarães J. H. 1971.Family Tachinidae (Larvaevoridae). A Catalogue of the Diptera of the Americaas South of the United States. São Paulo vol. 104, 1-333 pp.

Han H. Y., Choi D. S., Byun H.W., Lim O. Y. \& LeE H. S. 2004. A collection report of the Diptera (Insecta) from Mt. Gyemyeong, Chungju-si. Korean Journal of Nature Conservation. 2 (1-2): 123-131. [in Korean].

HAn H. Y., SuK S. W., LeE Y. B. \& LeE H. S. 2014. National List of Species of Korea [Insect] (Diptera II). National Institute of Biological Resources. Incheon. I-268 pp.

Herting B. \& Dely-Draskovits A. 1993. Family Tachinidae. In Soós Á. (ed.), Catalogue of Palaearctic Diptera, Anthomyiidae Tachinidae. Hungarian Natural History Museum. Vol.13, pp. 118-458, Budapest, 624 pp.

HIRASHIMA Y. 1989. Check List of Japanese Insects II. Pp: 541-1088. Fukuoka Entomological Laboratory, Faculty of Agiculture, Kyushu University and Japan Wild Life Research Center. [in Japanese].

KIM J. I. 1981. The faunistic study on the insects from Sudong-myeon, Namyangju-gun, Gyeonggi-do, Bulletin of the Korean Association for Conservation of Nature 3: 329-367. [in Korean].

KIM C. W. \& KIM J. I. 1971. Report on the preliminary survay of Mt. Odae and Sohgumgang, Chunghak-dong (Insects). Report of the Korean Association for Conservation of Nature 4: 139-173. [in Korean].

KIM C.W. \& KIM J. I. 1972. Insect fauna of Mt. Daedun, Haenam-gun. Report of the Korean Association for Conservation of Nature 6: 189-200. [in Korean].

KiM C.W. \& NAM S. H. 1978. Insect fauna of Imgye-myeon area in summer season. Report of the Korean Association for Conservation of Nature 13: 125-142. [In Korean].

LEE C. E. \& KWON Y. J. 1981. On the insect fauna of Isl. Ulreung and Dogdo in Korea. Report of the Korean Association for Conservation of Nature 19: 139-179. [In Korean].

LEE H.P., KiM O.S. \& KiM J. T. 1981. Studies on natura enemie parasiting overwinterig fall-webworm pupae, Hyphantria cunea Drury and the way of protection for parasitoids. Korean Journal of Entomology 11 (2): 11-16. [In Korean].

LEE H. S. \& HAN H. Y. 2007. A redescription of Pseudogonia rufifrons (Wiedemann) (Diptera: Tachinidae) in Korea. Journal of Asia-Pacific Entomology 10 (2): 103-107.

LEE H. S. \& HAN H. Y. 2008. A taxonomic study of the genus Frontina Meigen (Diptera: Tachinidae) in Korea. Journal of Asia-Pacific Entomology 11: 137-143.

LEE H. S. \& HAN H. Y. 2010. A systematic revision of the genus Gonia Meigen (Diptera: Tachinidae) in Korea. Animal Cells and Systems 14 (3): 175-195.

LiU Y. Z., Chao C. M., Li L. F., Zhou S. X., Wang H. X. \& Hang W. J. 1998. Fauna of Tachinidae from Shanxi Province, China. Science Press, Beijing, X+378 pp. +11 pls. [In Chinese].

MroczKowsKi M. 1972. Field Investigations in the Democratic People's Republic of Korea by staff members of the Institute of Zoology of the Polish Academy of Sciences. Fragmenta Faunistica, Warszawa, 18: 313-331.

Nakayama S.1929. A list of more important injurious insects in China. Bull. Agr. Exp. Stat., Gov. Chosen 4 (5): $261-$ 300. [In Japanese]

O’Hara J. E., Shima H. \& Zhang C. T. 2009. Annotated Catalogue of the Tachinidae (Insecta: Diptera) of China. Zootaxa 2190: 1-236.

O'Hara J. E. \& Wood M. 2004. Catalogue of the Tachinidae (Diptera) of America north of Mexico. Memoires on Entomology, International 18. iv $+410 \mathrm{pp}$.

Paek M. K., Hwang J. M., Jung K. S., Kim T. W., Kim M. C., Lee Y. I., Chao Y. B., Park S. W., Lee H. S., Ku D. S. et al. 2010. Checklist of Korean Insects. Nature and Ecology, Academic Series 2, Seul, 598 pp. [In Korean]

Park H. C., Park Y. C., Hong O. K. \& Chao S. Y. 1996. Parasitoids of the aphidophagous ladybeetles, Harmonia axyridis (Pallas) (Coleoptera: Coccinellidae) in Chuncheon areas, Korea. Korean Journal of Entomology 26: 143147. [In Korean] 
Pemberton R. W., Lee J. H., Reed D. K., Carlson R. W. \& Han H. Y. 1993. Natural enemies of the Asian gypsy moth (Lepidoptera: Lymantridae) in South Korea. Annals of the Entomological Society of America 86 (4): $423-440$.

Richter V. A. 2004. Fam. Tachinidae - tachinids. In: Sidorenko V.S. (ed.), Key to the insects of Russian Far East. Vol. VI. Diptera and Siphonaptera. Part 3 pp. 148-398. Vladivostk, Dal'nauka. 657 pp. [In Russian].

SHIMA H. 1968. Study on the Japanese Calocarcelia Townsend and Eucarcelia Baranov (Diptera: Tachinidae). Journal of the Faculty of Agriculture. Kyushu University 14: 507-513.

SHImA H. 1980. Study on the tribe Blondeliini from Japan (Diptera, Tachinidae) III, Descriptions of a new genus and two new species from Japan, Korea and Nepal, with notes on Drinomyia bicoloripes (Mesnil). Kontyû 48: 259266.

SHimA H. 1983. Study on the tribe Blondeliini from Japan (Diptera, Tachinidae). IV. A revision of the genus Vibrissina Rondani. Kontyû 51: 635-646.

Shima H. 1984. Study on the tribe Blondeliini from Japan (Diptera, Tachinidae). V. The genus Blondelia RobineauDesvoidy and Compsilura Bouché. Kontyû 52: 540-552.

SHima H. 1988. Some remarkable new species of Tachinidae (Diptera) from Japan and the Indo-Australian Region. Bulletin of the Kitakyushu Museum of Natural History 8: 1-37.

SHima H. 1996. A systematic study of the tribe Winthemiini from Japan. Beitrãge zur Entomologie 46: 169-235.

SHima H. 1999. Host - parasitic catalog of Japanese Tachinidae (Diptera). Makunagi/Acta Dipterologica 1 (Suppl.): $1-108$.

Shima H. 2006. A host-parasitic catalog of Tachinidae (Diptera) of Japan. Makunagi Acta Dipterologica Supplement 2: $1-177$.

Shima H. 2014. Family Tachinidae. In: NAKAmura, T., SAiguSA, T. \& SuwA, M. (eds.), Catalogue of the insects of Japan. Volume 8. Diptera. Part 2. Brachycera Schizophora, pp: 832-882. Entomological Society of Japan, Fukuoka, XIV + 562 pp.

Tachi T. \& Shima H. 2006. Systematic study of the genus Phorinia Robineau-Desvoidy of the Palaearctic, Oriental and Oceanian regions (Diptera: Tachinidae). Invertebrate Systematics 20: 255-287.

TSChORSNig H. P. \& Richter V. A. 1998. 3.54. Family Tachinidae. In: PAPP L. \& DARVAS B. (eds.). Contributions to a Manual of Palaearctic Diptera (with special references to flies of economic importance). Volume 3 . Higher Brachycera, pp. 691-827. Science Herald, Budapest. 880 pp.

ZIEGLER J. \& SHIMA H. 1996.Tachinid flies of the Ussuri area (Diptera: Tachinidae. Beiträge zur Entomologie 46: 379-478.

\section{STRESZCZENIE}

\section{[Stan wiedzy o faunie rączyc (Diptera, Tachinidae) Azji Wschodniej, z uwzględnieniem nowych danych z Korei Pólnocnej. Część V. Exoristinae]}

Opracowanie stanowi kontynuację badań nad fauną rączyc Korei Północnej. Materiał został zebrany przez pracowników Intytutu Zoologii PAN w Warszawie w latach 1959-1990, podczas sześciu wypraw do Korei Północnej. Muchówki były zbierane głównie siatką entomologiczną lub czerpakiem, a tylko w dwu ostatnich ekspedycjach zastosowano również żółte pułapki Moerick’a. Podrodzina Exoristinae jest największą wśród rączyc, w Palaeartyce dotychczas stwierdzono ponad 700 gatunków.

Zgromadzny materiał Exoristinae liczy 31 okazów, jest on jednak dość zróżnicowany. W Korei Północnej w obrębie opracowanej podrodziny znaleziono 24 gatunki z 20 rodzajów. Aktualna lista gatunków Exoristinae z Korei została uzupełniona przez 43 gatunki wymienione wcześniej przez innych autorów.

Obecnie z całej Korei znane są 72 gatunki zaliczane do 43 rodzajów, w tym 18 gatunków Exoristinae wymienionych jest $\mathrm{z}$ tego kraju po raz pierwszy. Muchówki z omawianej podrodziny w stadium larwalnym są zwykle endoparazytoidami: Lepidoptera, Coleoptera i Hymenoptera, ale również owady z rzędów: Dermaptera, Manthodea, Orthoptera i Phasmida są wymieniane jako żywiciele tych rączyc. 